ORIGINAL ARTICLE

\title{
Suppressive effects of breast milk on oxidative DNA damage in very low birthweight infants
}

\author{
H Shoji, T Shimizu, K Shinohara, S Oguchi, S Shiga, Y Yamashiro
}

\begin{abstract}
See end of article for authors' affiliations ....................

Correspondence to: Dr Shimizu, Department of Pediatrics, Juntendo University, School of Medicine, 2-1-1, Hongo, Bunkyo-ku, Tokyo, 1130033, Japan;

tsimizu†@aol.com
\end{abstract}

Accepted 10 March 2003
Arch Dis Child Fetal Neonatal Ed 2004;89:F136-F138. doi: 10.1136/adc.2002.018390

Background: Human milk contains many kinds of antioxidant and is considered to prevent diseases mediated by oxygen free radicals in very low birthweight (VLBW) infants.

Aims: To examine the antioxidant effects of breast milk in VLBW infants by determining urinary 8hydroxydeoxyguanosine $(8-\mathrm{OHdG})$ excretion, which is known to be a non-invasive marker for in vivo oxidative DNA damage.

Methods: Urinary 8-OHdG concentrations were measured in 15 breast fed and 14 formula fed VLBW infants at 2, 7, 14, and 28 days of age.

Results: Urinary 8-OHdG excretion at 14 and 28 days of age was significantly lower than at 2 and 7 days of age in the breast fed group, and significantly lower than in the formula fed group.

Conclusion: This is the first direct evidence of the antioxidant action of human milk in VLBW infants.
$\mathrm{P}$ remature infants are exposed to many possible sources of oxygen free radical production including high concentrations of inspired oxygen, frequent alterations in blood flow to major organs, and inflammation with accumulation of neutrophils and macrophages. ${ }^{12}$ Moreover, premature infants are known to have a poorly developed antioxidant system and may be at increased risk of radical damage. ${ }^{3-5}$ An imbalance between oxidant generating systems and antioxidants in very low birthweight (VLBW) infants is implicated in the pathogenesis of the major complications of prematurity, including necrotising enterocolitis (NEC), ${ }^{6}$ chronic lung disease (CLD), ${ }^{7}$ retinopathy of prematurity (ROP), ${ }^{8}$ and intraventricular haemorrhage (IVH). ${ }^{9}$

Human milk has many enzymatic and non-enzymatic antioxidant constituents, including superoxide dismutase, glutathione peroxidase, catalase, vitamins $\mathrm{E}$ and $\mathrm{A}$, and $\beta$-carotene, which may protect against the development of complications induced by oxygen free radical in infants. ${ }^{10-12}$ Breast feeding has been associated with low rates of a variety of illnesses in premature infants, including NEC, ${ }^{13}$ respiratory disease, ${ }^{14}$ and ROP, ${ }^{15}$ although there are no direct data establishing an antioxidant action of breast milk in infants.

Various methods have been established to evaluate oxidative stress and oxidative tissue damage, but accurate and reliable measurement of oxygen free radical activity is not easy. 8-Hydroxydeoxyguanosine ( 8 -OHdG) is accepted as a sensitive marker for oxidative DNA damage. ${ }^{16}{ }^{17}$ On hydroxylation, it is excised by constitutive enzymatic repair systems and subsequently excreted intact in the urine. Therefore, urinary 8-OHdG excretion can serve as a noninvasive marker for in vivo oxidative DNA damage. ${ }^{18}$ Recent studies have described the relation between prematurity and urinary 8-OHdG concentrations in low birthweight infants. ${ }^{19}{ }^{20}$ However, there have been no previous studies to compare urinary 8-OHdG excretion between breast fed and formula fed VLBW infants. If breast milk has a suppressive effect on oxidative tissue damage in VLBW infants, breast feeding should improve the outcome of VLBW infants. Therefore, to examine the antioxidant effects of breast milk in VLBW infants, we measured urinary 8 -OHdG concentrations in breast fed and formula fed VLBW infants at 2, 7, 14, and 28 days of age.

\section{MATERIALS AND METHODS \\ Patients}

Infants were eligible for this study if they had a birth weight less than $1500 \mathrm{~g}$, were born in or transported to the neonatal intensive care unit at Juntendo Izu Nagaoka Hospital between April 2000 and August 2001, and had no major congenital abnormalities. This study was approved by the institutional review board, and written informed consent was obtained from all guardians before inclusion of all subjects in the study.

The breast fed group included 15 infants (eight boys and seven girls; mean (SD) gestational age and birth weight 29.2 (2.3) weeks and 1231 (298) g) who received more than $90 \%$ of their intake as breast milk. The formula fed group included 14 infants (eight boys, six girls; 28.7 (2.0) weeks and $1182(281) \mathrm{g}$ ) who received more than $90 \%$ of their intake as commercial formula for premature infants. All of the subjects started feeding at 12-72 h after birth, and in most cases feeding started before the first sampling for urinary 8-OHdG measurement. There were no significant differences in the mean gestational age, birth weight, periods of oxygen therapy, phototherapy, and parenteral lipid infusion between the breast fed and formula fed groups (table 1).

\section{Measurement of urinary 8-OHdG}

Spot urine samples were collected in the morning at 2, 7, 14, and 28 days of age. They were then stored at $-20^{\circ} \mathrm{C}$ until the assay. The concentration of 8-OHdG was determined using a commercially available competitive enzyme linked immunosorbent assay (ELISA) kit (8-OHdG check; Japan Institute for the Control of Aging, Shizuoka, Japan). The specificity of the assay was established, ${ }^{21}$ and the determination range was 0.64-2000 ng/ml. Urinary 8-OHdG excretion was expressed as a creatinine ratio.

Abbreviations: 8-OHdG, 8-hydroxydeoxyguanosine; VLBW, very low birth weight; NEC, necrotising enterocolitis; CLD, chronic lung disease; ROP, retinopathy of prematurity; IVH, intraventricular haemorrhage;

ELISA, enzyme linked immunosorbent assay 
Table 1 Group characteristics of breast fed and formula fed infants

\begin{tabular}{|c|c|c|}
\hline & $\begin{array}{l}\text { Breast fed } \\
(n=15)\end{array}$ & $\begin{array}{l}\text { Formula fed } \\
(n=14)\end{array}$ \\
\hline Gestational age (weeks) & $29.2(2.3)$ & $28.7(2.0)$ \\
\hline Birth weight $(\mathrm{g})$ & $1231(298)$ & $1182(281)$ \\
\hline Sex (male:female) & $8: 7$ & $8: 6$ \\
\hline Antenatal steroids & $3 / 15$ & $2 / 14$ \\
\hline Five minute Apgar $<6$ & $4 / 15$ & $3 / 14$ \\
\hline Period of phototherapy (hours) & $68.5(62.4)$ & $72.5(68.5)$ \\
\hline Period of ventilation (days) & $15.6(14.7)$ & $14.1(12.5)$ \\
\hline Period of oxygen therapy (days) & $43.3(40.4)$ & $52.3(50.9)$ \\
\hline $\begin{array}{l}\text { Period of parenteral nutrition } \\
\text { (days) }\end{array}$ & $12.3(3.1)$ & $14.4(3.9)$ \\
\hline $\begin{array}{l}\text { Period of parenteral intralipid } \\
\text { (days) }\end{array}$ & $6.2(2.1)$ & $6.3(2.6)$ \\
\hline Retinopathy of prematurity & $4 / 15$ & $2 / 14$ \\
\hline Intraventricular haemorrhage & $1 / 15$ & $2 / 14$ \\
\hline Chronic lung disease & $2 / 15$ & $2 / 14$ \\
\hline Necrotising enterocolitis & $1 / 15$ & $0 / 14$ \\
\hline
\end{tabular}

Where appropriate, values are mean (SD).

\section{Statistical analysis}

Differences between the different ages were tested by the Wilcoxon signed rank test, and those between the two groups by the Mann-Whitney $U$ test and Fisher's exact test. $p<0.05$ was considered significant.

\section{RESULTS}

In the breast fed group, urinary 8-OHdG excretion at 14 and 28 days of age was significantly lower $(p<0.01)$ than that at 2 and 7 days of age (fig 1). In the formula fed group, there were no significant differences in urinary 8 -OHdG excretion observed at 2, 7, 14, and 28 days of age. Urinary 8-OHdG excretion at 14 and 28 days of age in the breast fed group was significantly lower $(\mathrm{p}<0.01)$ than that in the formula fed group.

There were no significant differences in the incidence of ROP, IVH, CLD, and NEC between the two groups (table 1).

\section{DISCUSSION}

Our results suggest that oxidative DNA damage is considerably more suppressed in breast fed VLBW infants than in formula fed VLBW infants at 14 and 28 days of age. Although human milk contains many kinds of antioxidant and has long been thought to prevent diseases mediated by oxygen free radicals in VLBW infants, ${ }^{10-12}$ our study is the first to present direct evidence of this antioxidant action by measuring urinary 8-OHdG excretion. Although the measurement of lipid peroxide concentrations using the malondialdehydethiobarbituric acid assay is the most widely used method of

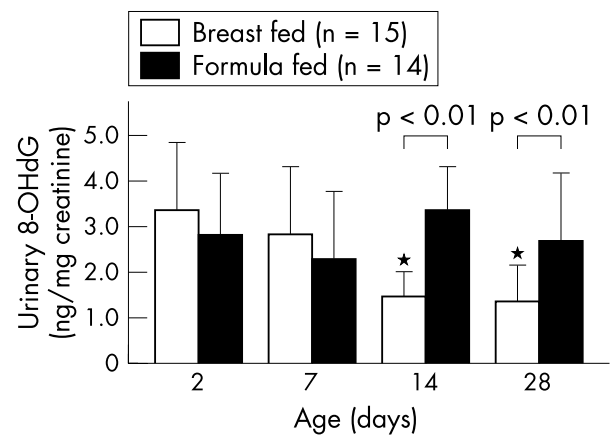

Figure 1 Change in urinary 8-hydroxydeoxyguanosine (8-OHdG) excretion at $2,7,14$, and 28 days of age in breast fed and formula fed very low birthweight infants. Values are mean (SD). ${ }^{*} p<0.01$ compared with 2 and 7 days. measuring free radical activity, ${ }^{22-24}$ its accuracy in measuring radical activity and the resultant lipid peroxidation may be compromised by its indirectness and susceptibility to contamination by other thiobarbituric acid reactive species. Damaged DNA is repaired by non-specific endonucleases and specific glycosylases in vivo, and eliminated oxidised nucleotides are finally excreted into the urine as 8-OHdG. 8-OHdG concentrations have been measured by high performance liquid chromatography with electrochemical detection and gas chromatography-mass spectrometry, even though the complicated extraction procedures are known to cause recovery problems. ${ }^{25}$ An ELISA based on monoclonal IgG has been developed for estimation of 8 -OHdG in urine samples. ${ }^{21}$ This method has made it easier to measure urinary 8-OHdG. ${ }^{26}$ Measurement of urinary 8-OHdG excretion is also considered to be a very sensitive biomarker of oxidative DNA damage in low birthweight infants. ${ }^{19}{ }^{20}$ It may also be useful as a true biomarker for free radical mediated diseases in VLBW infants, as well as a good index of the effects of antioxidant supplementation with agents such as selenium.

In this study, there were no significant differences in urinary 8-OHdG levels at 2 and 7 days of age between the breast fed and formula fed groups. There are many factors that can cause oxidative stress resulting in DNA damage in the early stages of life in VLBW infants, such as high concentrations of inspired oxygen, phototherapy, and intravenous lipid infusion. ${ }^{12}$ Therefore, a possible explanation for the lack of antioxidant effects of breast milk during the first week of life is that the oxidative stress caused by these factors was too strong for the DNA damage to be suppressed. Another possible explanation for the lack of difference in urinary 8 -OHdG concentrations between breast fed and formula fed infants at 2 days of age is that some of the infants had not yet started feeding at 2 days of age. It may also be related to the lack of any significant differences between the two groups with respect to the incidence of chronic complications such as ROP, IVH, CLD, and NEC. Inflammatory changes are probable precursors of these complications, and they are known to occur in the earliest stages of life. ${ }^{127}$ A randomised controlled trial comparing the outcomes of breast fed and formula fed VLBW infants should help to explain this.

The exact antioxidative mechanisms of breast feeding in VLBW infants remain unclear. A possible mechanism is the effects of the antioxidants in breast milk on the various oxidative stresses and subsequent DNA damage. Human milk is known to contain many kinds of antioxidant, ${ }^{10-12}$ but little is known about their stability in the gastrointestinal tract, their direct effects on the mucosa, their absorption from the intestine, and their effects after absorption into the blood. To elucidate the actual way in which antioxidants in breast milk protect against oxidative stress in VLBW infants, we need to examine their kinetics and effects. Another possible mechanism is promotion of the endogenous antioxidant activities of superoxide dismutase, glutathione peroxidase, and other enzymes by various substances in breast milk. These direct and indirect effects may explain the difference in urinary 8OHdG excretion between breast fed and formula fed VLBW infants in this study.

In conclusion, measurement of urinary $8-\mathrm{OHdG}$ excretion is useful for the assessment of oxidative DNA damage in VLBW infants. Human milk can suppress oxidative stress and oxidative DNA damage after 14 days of age.

\section{ACKNOWLEDGEMENTS}

We thank Kazuo Dairiki (Meiji Dairies Corporation) for valuable assistance in measuring the concentrations of urinary 8-OHdG. 


\section{Authors' affiliations}

H Shoii, T Shimizu, K Shinohara, S Oguchi, S Shiga, Y Yamashiro, Department of Paediatrics, Juntendo University School of Medicine, Tokyo, Japan

\section{REFERENCES}

1 Saugstad OD. Mechanisms of tissue injury by oxygen radicals: implication for neonatal disease. Acta Paediatr 1996;85:1-4.

2 Groneck P, Speer CP. Inflammatory mediators and bronchopulmonary dysplasia. Arch Dis Child Fetal Neonatal Ed 1995;73:F1-3.

3 Pitkkanen OM, Hallman M, Anderson SM. Correlation of free radical-induced lipid peroxidation with outcome in very low birthweight infants. J Pediatr 1990;116:760-4.

4 Lindman JHN, van Zoergen-Grobben D, Schrijver J. The total free radical trapping ability of cord blood plasma in preterm and term babies. Pediatr Res 1989;26:20-4.

5 Sullivan JL, Newton RB. Serum antioxidant activity in neonates. Arch Dis Child 1988;63:748-57.

6 Okur H, Kucukaydin M, Kose K, et al. Hypoxia-induced necrotizing enterocolitis in the immature rat: the role of lipid peroxidation and management by vitamin E. J Pediatr Surg 1995;30:1416-19.

7 Saugstad OD. Bronchopulmonary dysplasia and oxidative stress: are we closer to an understanding of the pathogenesis of BPD? Acta Paediatr 1997:86:1277-82.

8 Muller DPR. Vitamin E therapy in retinopathy of prematurity. Eye 1992;6:221-5.

9 Kelly FJ. Free radical disorders of preterm infants. Br Med Bull 1993;49:668-78.

10 Lindmark-Mansson $\mathrm{H}$, Akesson B. Antioxidative factors in milk. Br J Nutr 2000;84:S103-10.

11 Goldman AS, Goldblum RM, Hanson LA. Anti-inflammatory systems in human milk. Adv Exp Med Biol 1990;262:69-76.

12 Buescher ES, Mcllherhan SM. Antioxidant properties of human colostrums. Pediatr Res 1988;24:14-19.

13 Lucas A, Cole TJ. Breast milk and neonatal necrotising enterocolitis. Lancet 1990;336:1519-23.

14 Watkins CJ, Leeder SR, Corkhill RT. The relationship between breast and bottle feeding and respiratory illness in the first year of life. J Epidemiol Community Health 1979;33:180-2.
15 Hylander MA, Strobino DM, Pezzullo JC, et al. Association of human milk feedings with a reduction in retinopathy of prematurity among very low birthweight infants. J Perinatol 2001;21:356-362.

16 Shigenaga MK, Ames BN. Assays for 8-hydroxy-2'-deoxyguanosine: a biomarker of in vivo oxidative DNA damage. Free Radic Biol Med $1991 ; 10: 211-16$.

17 Fraga CG, Shigenaga MK, Park JW, et al. Oxidative damage to DNA during aging: 8-hydroxy-2'-deoxyguanosine in rat organ DNA and urine. Proc Nat Acad Sci USA 1990;87:4533-7.

18 Lunec J, Herbert K, Blount S, et al. 8-Hydroxydeoxyguanosine. A marker of oxidative DNA damage in systemic lupus erythematosus. FEBS Lett 1994;348:131-8.

19 Matsubasa T, Uchino T, Karashima S, et al. Oxidative stress in very low birth weight infants as measured by urinary $8-\mathrm{OHdG}$. Free Radic Res 2002;36:189-93.

20 Drury JA, Jeffers G, Cooke RW. Urinary 8-hydroxydeoxyguanosine in infants and children. Free Radic Res 1998;28:423-4.

21 Toyokuni S, Tanaka T, Hattori Y, et al. Quantitative immunohistochemical determination of 8-hydroxy-2'-deoxyguanosine by a monoclonal antibody N45. 1: its application to ferric nitrilotriacetate-induced renal carcinogenesis model Lab Invest 1997;76:365-74.

22 Inder TE, Darlow BA, Sluis KB, et al. The correlation of elevated levels of an index of lipid peroxidation (MDA-TBA) with adverse outcome in the very low birthweight infant. Acta Paediatr 1996;85:1116-22.

23 Inder TE, Graham P, Sanderson K, et al. Lipid peroxidation as a measure of oxygen free radical damage in the very low birthweight infant. Arch Dis Child Fetal Neonatal Ed 1994:70:F107-11.

24 Winterbourn CC, Chan T, Buss $\mathrm{H}$, et al. Protein carbonyls and lipid peroxidation products as oxidation markers in preterm infant plasma: associations with chronic lung disease and retinopathy and effects of serenium supplementation. Pediatr Res 2000:48:84-90.

25 Loft S, Poulsen HE. Cancer risk and oxidative DNA damage in man. J Mol Med 1996;74:297-312.

26 Saito S, Yamauchi H, Hasui Y, et al. Quantitative determination of urinary 8hydroxydeoxyguanosine (8-OH-dg) by using ELISA. Res Commun Mol Pathol Pharmacol 2000; 107:39-44.

27 Murch SH, Costeloe K, Klein NJ, et al. Early production of macrophage inflammatory protein-1 alpha occurs in respiratory distress syndrome and is associated with poor outcome. Pediatr Res 1996;40:490-7. 